\title{
Contents of Volume 1
}

\author{
Preface to the second edition — IX
}

Preface to the first edition $-\mathbf{X I}$

1 Heuristics and history - 1

$1.1 \quad$ Feynman path integrals and Feynman-Kac formulae -1

1.2 Plan and scope of the second edition -5

2 Brownian motion - 9

2.1 Concepts and facts of general measure theory and probability -9

2.1.1 Elements of general measure theory - 9

2.1.2 Probability measures and limit theorems - 16

2.1.3 Random variables - 29

2.1.4 Conditional expectation and regular conditional probability measures - 38

$2.2 \quad$ Random processes $-\mathbf{4 5}$

2.2.1 Basic concepts and facts -45

2.2.2 Martingale properties $-\mathbf{5 0}$

2.2.3 Stopping times and optional sampling - $\mathbf{5 3}$

2.2.4 Markov properties — 67

2.2.5 Feller transition kernels and generators - $\mathbf{7 2}$

2.2.6 Invariant measures -74

2.3 Brownian motion and Wiener measure - 77

2.3.1 Construction of Brownian motion - 77

2.3.2 Two-sided Brownian motion - 84

2.3.3 Conditional Wiener measure - $\mathbf{8 8}$

2.3.4 Martingale properties of Brownian motion - $\mathbf{8 9}$

2.3.5 Markov properties of Brownian motion - 92

2.3.6 Local path properties of Brownian motion - 97

2.3.7 Global path properties of Brownian motion - 103

2.4 Stochastic calculus based on Brownian motion - 107

2.4.1 The classical integral and its extensions - 107

2.4.2 Stochastic integrals -108

2.4.3 Extension of stochastic integrals - $\mathbf{1 1 5}$

2.4.4 Itô formula - 119

2.4.5 Stochastic differential equations - 128

2.4.6 Brownian bridge -134

2.4.7 Weak solution and time change - 136

2.4.8 Girsanov theorem and Cameron-Martin formula - 140 
3 Lévy processes - 143

3.1 Lévy processes and the Lévy-Khintchine formula - 143

3.1.1 Infinitely divisible random variables - 143

3.1.2 Lévy-Khintchine formula - 149

3.1.3 Lévy processes - 154

3.1.4 Martingale properties of Lévy processes - 160

3.1.5 Markov properties of Lévy processes - 161

3.2 Sample path properties of Lévy processes - 165

3.2.1 Càdlàg version -165

3.2.2 Two-sided Lévy processes -169

3.3 Random measures and Lévy-Itô decomposition - $\mathbf{1 7 8}$

3.3.1 Poisson random measures $-\mathbf{1 7 8}$

3.3.2 Lévy-Itô decomposition — 186

3.4 Itô formula for semimartingales - 188

3.4.1 Point processes -188

3.4.2 Itô formula for semimartingales - 194

3.5

3.5 .1 Exponentials of Lévy processes and recurrence properties 201

3.5.2 Capacitary measures -203

3.5.3 Recurrence properties of Lévy processes - 204

3.6

3.6.1 Subordinators and Bernstein functions - 206

3.6.2 Bernstein functions -209

$4 \quad$ Feynman-Kac formulae -217

4.1 Schrödinger semigroups - 217

4.1.1 Schrödinger equation and path integral solutions - 217

4.1.2 Linear operators and their spectra - 218

4.1.3 Spectral resolution - 223

4.1.4 Compact operators and trace ideals - 227

4.1.5 Schrödinger operators -232

4.1.6 Schrödinger operators through quadratic forms - 236

4.1.7 Confining potentials and decaying potentials -239

4.1.8 Strongly continuous operator semigroups $-\mathbf{2 4 3}$

4.2 Feynman-Kac formula for Schrödinger operators - 246

4.2.1 Bounded smooth external potentials - 246

4.2.2 Derivation through the Trotter product formula $-\mathbf{2 4 9}$

4.2.3 Kato-class potentials $-\mathbf{2 5 1}$

4.2.4 Feynman-Kac formula for Kato-decomposable potentials -264

4.3 Properties of Schrödinger operators and semigroups $-\mathbf{2 7 0}$

4.3.1 Kernel of the Schrödinger semigroup $-\mathbf{2 7 0}$

4.3.2 Positivity improving and uniqueness of ground state $-\mathbf{2 7 1}$ 
4.3.3 Degenerate ground state and Klauder phenomenon - $\mathbf{2 7 5}$

4.3.4 Existence and non-existence of ground states - 277

4.3.5 Sojourn times and existence of bound states $-\mathbf{2 8 2}$

4.3.6 The number of eigenfunctions with negative eigenvalues - 289

4.3.7 Application to canonical commutation relations - $\mathbf{3 0 7}$

4.3.8 Exponential decay of eigenfunctions - 314

4.4 Feynman-Kac formula for Schrödinger operators with vector potentials - $\mathbf{3 2 0}$

4.4.1 Feynman-Kac-Itô formula - $\mathbf{3 2 0}$

4.4.2 Alternative proof of the Feynman-Kac-Itô formula - $\mathbf{3 2 4}$

4.4.3 Extension to singular external and vector potentials - 327

4.4.4 Kato-class potentials and $L^{p}-L^{q}$ boundedness -333

4.5 Feynman-Kac formula for unbounded semigroups and Stark effect - 335

4.6 Feynman-Kac formula for relativistic Schrödinger operators - 339

4.6.1 Relativistic Schrödinger operator - 339

4.6.2 Relativistic Kato-class potentials - $\mathbf{3 4 4}$

4.6.3 Decay of eigenfunctions - 351

4.6.4 Non-relativistic limit -356

4.7 Feynman-Kac formula for Schrödinger operators with spin - 359

4.7.1 Schrödinger operators with spin $\frac{1}{2}-359$

4.7.2 A jump process - $\mathbf{3 6 1}$

4.7.3 Feynman-Kac formula for the jump process - $\mathbf{3 6 3}$

4.7.4 Extension to singular external potentials and singular vector potentials - 367

4.7.5 Decay of eigenfunctions and martingale properties - $\mathbf{3 7 1}$

4.8 Feynman-Kac formula for relativistic Schrödinger operators with spin - 375

4.8.1 Relativistic Schrödinger operator with spin $\frac{1}{2}-\mathbf{3 7 5}$

4.8.2 Martingale properties - 381

4.8.3 Decay of eigenfunctions - 384

4.9 Feynman-Kac formula for nonlocal Schrödinger operators - $\mathbf{3 8 8}$

4.9.1 Nonlocal Schrödinger operators - 388

4.9.2 Vector potentials - $\mathbf{3 8 9}$

4.9.3 $\quad \Psi$-Kato-class potentials -392

4.9.4 Fractional Kato-class potentials - $\mathbf{4 0 1}$

4.9.5 Generalized spin - $\mathbf{4 0 6}$

4.9.6 Recurrence properties and existence of bound states - $\mathbf{4 1 2}$

4.9.7 The number of eigenfunctions with negative eigenvalues - 413

4.9.8 Decay of eigenfunctions - $\mathbf{4 2 3}$

4.9.9 Massless relativistic harmonic oscillator $-\mathbf{4 3 2}$

4.9.10 Embedded eigenvalues — 436 
$5 \quad$ Gibbs measures associated with Feynman-Kac semigroups - 449

5.1 Ground state transform and related processes - 449

5.1.1 Ground state transform and the intrinsic semigroup - 449

5.1.2 Ground state-transformed processes as solutions of SDE -454

5.1.3 $P(\phi)_{1}$-processes with continuous paths -458

5.1.4 Dirichlet principle -464

5.1.5 Mehler's formula -467

5.1.6 $P(\phi)_{1}$-processes with càdlàg paths - 476

$5.2 \quad$ Gibbs measures on path space $-\mathbf{4 8 1}$

5.2.1 From Feynman-Kac formulae to Gibbs measures - 481

5.2.2 Gibbs measures on Brownian paths - 485

5.2.3 Gibbs measures on càdlàg paths -492

5.3 Gibbs measures for external potentials - 494

5.3.1 Existence -494

5.3.2 Uniqueness -497

5.4 Gibbs measures for external and pair interaction potentials: direct method - $\mathbf{5 0 3}$

5.5 Gibbs measures for external and pair interaction potentials: cluster expansion - $\mathbf{5 1 1}$

5.5.1 Cluster representation $-\mathbf{5 1 1}$

5.5.2 Basic estimates and convergence of cluster expansion - $\mathbf{5 1 6}$

5.5.3 Further properties of the Gibbs measure - $\mathbf{5 1 8}$

$6 \quad$ Notes and references -521

Notes to the Preface $-\mathbf{5 2 1}$

Notes to Chapter $1-\mathbf{5 2 2}$

Notes to Chapter $2-\mathbf{5 2 2}$

Notes to Chapter $3-\mathbf{5 2 5}$

Notes to Chapter $4-\mathbf{5 2 6}$

Notes to Chapter $5-\mathbf{5 3 5}$

Bibliography — 539

Index -553 